\title{
Mathematical Model of Landslides with Curved Sliding Plane Using Fracture Criterion $(\tau-\sigma)$ of Solid Soil-Rock System
}

\author{
Ikah Ning Prasetiowati Permanasari1*, Lilik Hendrajaya², Gunawan Handayani² \\ ${ }^{1}$ Departemen of Physics, Institut Teknologi Sumatera, Lampung 35365, Indonesia \\ ${ }^{2}$ Departemen of Physics, Institut Teknologi Bandung, Bandung 40132, Indonesia \\ *Corresponding author: ikahning@fi.itera.ac.id
}

\begin{abstract} friction coefficient $A$.

Keywords

Curving Fracture Plane, Friction Coefficient, Landslide, Mohr Circle, Stress
\end{abstract}

Alluvial cliff with high slope and step has been known to cause curving sliding plane. This phenomenon is adopted as a knowledge, but it has never been studied theoretically. In this paper, mathematics derivation phenomenon is proposed using special model of fracture criterion $\tau-\sigma$. The quantity of $\tau$ is A $\sigma^{\frac{1}{2}}$ with A is a friction coefficient of stress $\sigma^{\frac{1}{2}}$. The failure model was substituted to the corresponding Mohr circle resulting the expected curve. The curve was used to measure the observed field profile to obtain the

Received: 26 July 2021, Accepted: 14 October 2021

https://doi.org/10.26554/sti.2022.7.1.1-8

\section{INTRODUCTION}

Landslide is the most common disaster in steep slope area and it is particularly occurred in rainy season (Fan et al., 2017; Permanasari et al., 2020; Gariano and Guzzetti, 2016; Alvioli et al., 2014). Landslide defined generally as the movement of rock, debris and soil down to slope driven by gravity and preconditioned by the landform (Permanasari et al., 2020). The topic of landslides is widely studied from various disciplines (Shanmugam and Wang, 2015). But, reports and publication of landslides mostly described the process and qualitatively analyzed the role of water and clays which causes less friction coefficient along the sloping rock body (Wu et al., 2017; Saito et al., 2017; Wu et al., 2016; Alsubal et al., 2019). Other studies mostly only do landslide dynamics modeling, perform simulations to gain knowledge of how the transport process in landslides (Li et al., 2012; Dai et al., 2014; Shi et al., 2016). To the best of our knowledge, the quantitative analysis of parameters of the before and after disaster has not been studied through mechanism model (De-Blasio, 2011; Zhang et al., 2016; Bin et al., 2020; Padmaja et al., 2021). Therefore, in this paper we developed the mechanism model to obtain the parameters of the landslide disaster using fracture criterion $\tau-\sigma$. The procedure to develop the model is as follow:

1. Strat by observing the field phenomena

2. Constructing theoretical models by applying physical laws in equations and with rigorous calculus to produce theo- retical behavior.

3. If the theoretical behavior forms geometry that corresponds to the the actual phenomena (field data), then the theoretical equations can be used to measure field parameters.

4. Elaborating this theory description with all results comprehensively, to have further results for mitigation.

In this paper, the authors introduce a comprehensive model of a cliff with height $\mathrm{H}$, under heavy rainfall that saturates the soil-rock system and produce curved sliding plane. The model is two dimensional $(x-y)$, due to failure is determined by two principal stresses namely maximum stress $\left(\sigma_{\max }\right)$ and minimum stress $\left(\sigma_{\min }\right)$ (Figure 1).

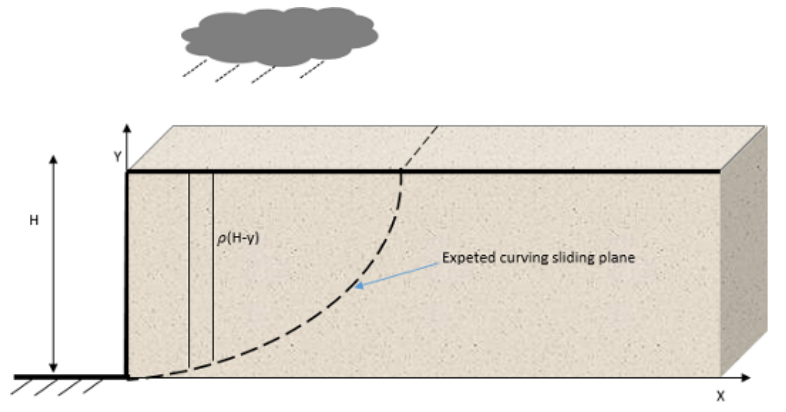

Figure 1. Sketch of a Step Function Cliff 


\section{THE MODEL}

The model is a continuum soil-rock system. It is sliced into a slab dx at point $x$ and at position $y$, where the expected fractures occur.

\subsection{Flow of Thought Towards Mechanism: Mathematical Physics Model of Curved Collapse}

2.1.1 The Intersection of Mohr's Circle and $\tau=f(\sigma)$ In the Mohr-Coloumb theory it is explained that the cracking process in solids occurs due to a combination of the critical state of normal stress and shear stress acting on the material (Figure 2) (Das, 2016; Labuz and Zhang, 2012).
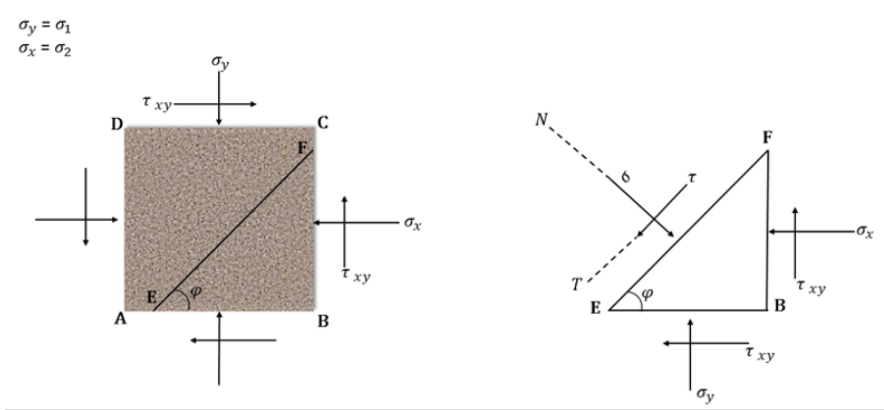

Figure 2. Soil Elements with Normal Stress and Shear Stress Acting on The Element (a), Force Diagram on The EFB (b)

From the rules of mechanics, what is added according to the direction is the force, not the stress (Wesley., 2013). This means that stress must be multiplied by the area of the field, as an Equation 1 and 2 below:

$$
\overline{E B}=\overline{E F} \cos \varphi
$$

$$
\overline{F B}=\overline{E F} \sin \varphi
$$

By adding up the components of the force acting in the direction $\mathrm{N}$ and $\mathrm{T}$, we get:

$$
\sigma(\overline{E F})=\sigma_{x} \overline{E F} \sin ^{2} \varphi+\sigma_{y} \overline{E F} \cos ^{2} \varphi+2 \tau_{x y} \overline{E F} \sin \varphi \cos \varphi
$$

or

$$
\sigma=\sigma_{x} \sin ^{2} \varphi+\sigma_{y} \cos ^{2} \varphi+2 \tau_{x y} \sin \varphi \cos \varphi
$$

or

$$
\sigma=\frac{\sigma_{y}+\sigma_{x}}{2}+\frac{\sigma_{y}-\sigma_{x}}{2} \cos 2 \varphi+\tau_{x y} \sin 2 \varphi
$$

Then for the component $\tau$ :

$$
\tau=-\sigma_{x}(\overline{E F}) \sin \varphi \cos \varphi+\sigma_{y}(\overline{E F}) \sin \varphi \cos \varphi-
$$

$$
\tau_{x y}(\overline{E F}) \cos ^{2} \varphi+\tau_{x y}(\overline{E F}) \sin ^{2} \varphi
$$

or

$$
\tau=\sigma_{y} \sin \varphi \cos \varphi-\sigma_{x} \sin \varphi \cos \varphi-\tau_{x y}\left(\cos ^{2} \varphi-\sin ^{2} \varphi\right)
$$

or

$$
\tau=\frac{\tau_{y}-\tau_{x}}{2} \sin 2 \varphi-\tau_{x y} \cos 2 \varphi
$$

In the case where the planes $\mathrm{AB}$ and $\mathrm{AD}$ are major and minor planes, the normal stress and shear stress in the plane EF can be obtained by substituting $\tau_{x y}=0$ in equations 3 and 4 , where $\sigma_{y}=\sigma_{1}$ and $\sigma_{x}=\sigma_{3}$ so that we get value of $\sigma$ and $\tau$ as in Equation 5 and 6.

$$
\begin{aligned}
& \sigma=\frac{\sigma_{1}+\sigma_{3}}{2}+\frac{\sigma_{1}-\sigma_{3}}{2} \cos 2 \varphi \\
& \tau=\frac{\sigma_{1}-\sigma_{3}}{2} \sin 2 \varphi
\end{aligned}
$$

where $\sigma_{\max }=\sigma_{1}$ and $\sigma_{\min }=\sigma_{3}$ so that we get the value of $\sigma$ and $\tau$ as in Equation 7 and 8

$$
\begin{aligned}
& \sigma=\frac{\sigma_{\max }+\sigma_{\min }}{2}+\frac{\sigma_{\max }-\sigma_{\min }}{2} \cos 2 \varphi \\
& \tau=\frac{\sigma_{\max }-\sigma_{\min }}{2} \sin 2 \varphi
\end{aligned}
$$

then we substitute the two equations above,

$$
\cos ^{2} 2 \varphi+\cos ^{2} 2 \varphi=1
$$

then obtained, Equation 9 is a circle as a potential crack field.

$$
\left(\sigma^{2}-\frac{\sigma_{\max }+\sigma_{\min }}{2}\right)^{2}+\tau^{2}=\left(\frac{\sigma_{\max }-\sigma_{\min }}{2}\right)^{2}
$$

Based on Mohr's theory, a triaxial measurement method was developed to measure rock/soil properties by measuring the direction of cracks when the sample is pressed from three directions (Figure 3) (Lelovic and Vasovic , 2020; Helwany, 2020). Where the triaxial test is to provide an external force 


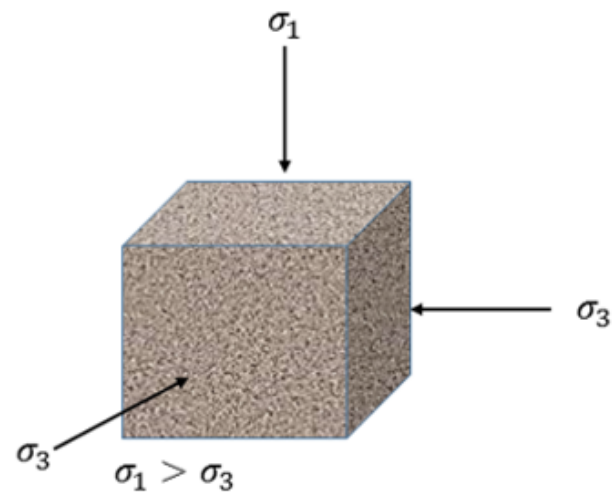

Figure 3. Triaxial Test Sample Illustration

or stress to produce cracks in rock or solid soil according to Mohr's criteria (Murthy, 2002).

By recording $\sigma_{\max }$ (vertical) and $\sigma_{\min }$ (horizontal) measurements of the angle between cracks and $\sigma_{\min }$ are carried out. Then by drawing on the $\tau-\sigma$ axis, namely drawing $\sigma_{\max }$ and $\sigma_{\min }$ and the center point of the circle, Mohr's circle can be drawn with $\frac{\sigma_{\max }-\sigma_{\min }}{2}$ as the radius. With several experiments with samples from the same place with different combinations of $\sigma_{\max }$ and $\sigma_{\min }$, the locus points of the curve $\tau=\mathrm{f}(\sigma)$. Because the measurement is made from small $\sigma_{\max }$ to increase, it will get one point of tangency for each experiment which is the tangent point of Mohr's circle on the candidate $\tau=\mathrm{f}(\sigma)$. From many experiments carried out for one type of sample, it is obtained on average using equation 10 below (Das, 2002):

$$
\tau=c+\sigma \tan \phi
$$

Where : $\mathrm{c}=$ cohesion $\phi=$ internal stress angle $\tau=$ shear stress $\sigma=$ normal stress

\subsection{2 $\left(\sigma_{\max }, \sigma_{\min }\right)$ and $\tau=f(\sigma)$ Model}

For the model $\sigma_{\text {max }}, \sigma_{\text {min }}$, on a collapsed cliff, the condition of almost collapse is used because it is saturated with water (Thompshon and Huppert, 2007), with the outer side of the cliff being subjected to $\sigma_{\min }=$ horisontal stress $=0$ because there is no external support and the vertical side is lithostatic pressure (hydrostatic on soil/rock) i.e. $\sigma_{\max }=$ verticalstress $=\rho g^{*}$ height . Because $\sigma_{\min }=0$ then Mohr's circle will pass through the center of the circle or pass through the point $(0,0)$ so that the outermost layer in a certain thickness will collapse first.

For the model $\tau=\mathrm{f}(\sigma)$ based on the fact that there will be an upward curved crack, then $\sigma_{\max }$ will decrease and the crack angle will also decrease. So from the condition $(0,0)$ where the angle $\varphi$ is large, it decreases to the right in the direction of $\tan \varphi=\mathrm{d} \tau / \mathrm{d} \sigma$, then the $\tau=\mathrm{f}(\sigma)$ curve is curved from a large to a small angle resembling a half-parabola open in the direction of an enlarged $\sigma$ (Figure 4)

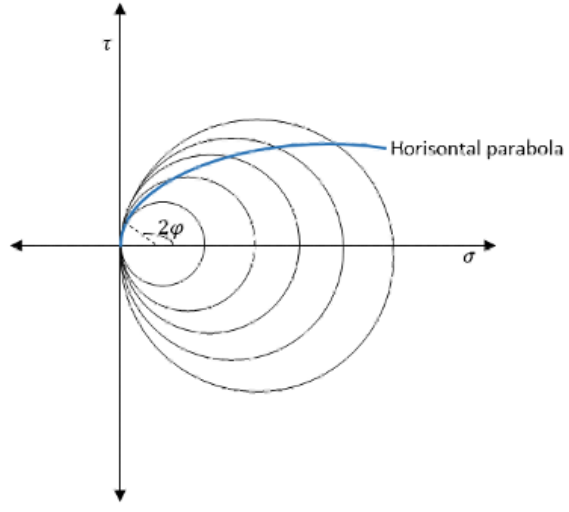

Figure 4. Mohr Intersection Point

It was observed that the crack angle $\varphi$ getting closer to the surface is getting bigger as the $\sigma_{\max }$ decreases. This phenomenon provides input that referring to the definition of the Mohr Coloumb diagram and the $\varphi$ or $2 \varphi$ relationship then, small $\varphi$ at high $\sigma_{\max }$ at the bottom and large $\varphi$ at small $\sigma_{\max }$ low at the top near the surface. From this situation, if it is connected, it becomes connected (envelope) the intersection point between Mohr's circle $\left(\sigma_{\max }=\rho g *\right.$ height and $\sigma_{\min }=$ 0 )which passes through $(0,0)$ will be in the form of a sketch of the curve that has a parabolic shape that opens in the direction of increasing $\sigma$.

Mathematically, the relationship between $\tau-\sigma$ can be written as Equation 11:

$$
\tau=\operatorname{constant} x \sigma^{\frac{1}{n}}
$$

Since Mohr's circle equation is a quadratic in $\tau$ and $\sigma$ and in order to be solved quadratic (parabolic model), we model as:

$$
\tau=A \tau^{\frac{1}{2}}
$$

2.2 Starting with $x=0, y=0$ i.e The Outer Slab Contacts with Free Surface (Air)

When water saturation increases on the cliff and reaches a condition immediate to liquefaction (Garry, 2004), the density of soil is $\rho$ at the bottom element suffers vertical lithostatic stress $=\rho g h$ with $g$ is the gravitational acceleration constant. At this outer wall, the bottom element has no stress (stress along $\mathrm{x} \cong 0$ ) thus, we have maximum stress $\sigma_{\max }=\rho g h$ and for any x and y $\sigma_{\max }=\rho g(H-y)$. The rupture or collapse process is the breaking of the slab $d x$. The cliff will collapse from left to right and resulting in the curving bottom part (Figure 5)

\subsection{Mohr Diagram Application}

Based on the conditions of $\sigma_{\max }=\rho g(H-y)$ and $\sigma_{\min }=0$, the Mohr circle can be drawn with the angle $\varphi$ is between $\sigma_{\text {min }}$ direction (x-axis) and the fracture plane (Figure 6). By following the geometry of $\tau$ (shear stress along fracture plane ) $-\sigma$ 


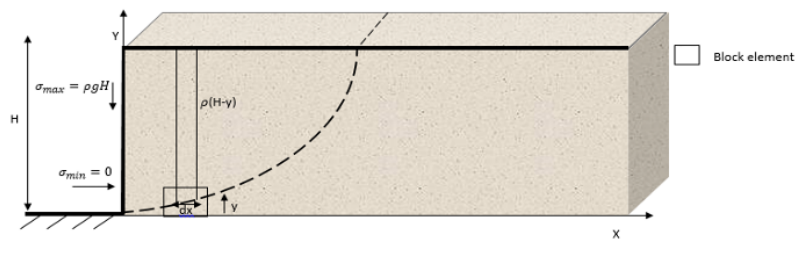

Figure 5. Step Function Model of Cliff Body and The Ruptured Goes from Left (Outer Slab) to Right

(normal stress on fracture plane) the Mohr's equation circle is is shown in Equation 13 :

$$
y=\left[\sigma-\frac{1}{2} \rho g(H-y)^{2}\right]+\tau^{2}=\left[\frac{1}{2} \rho g(H-y)^{2}\right]
$$

with

$$
\begin{aligned}
& \left.\sigma=\frac{1}{2} \rho g(H-y)+\frac{1}{2} \rho g(H-y) \cos \cos ^{2} 2 \varphi\right) \\
& \tau=\frac{1}{2} \rho g(H-y) \sin \sin 2 \varphi
\end{aligned}
$$

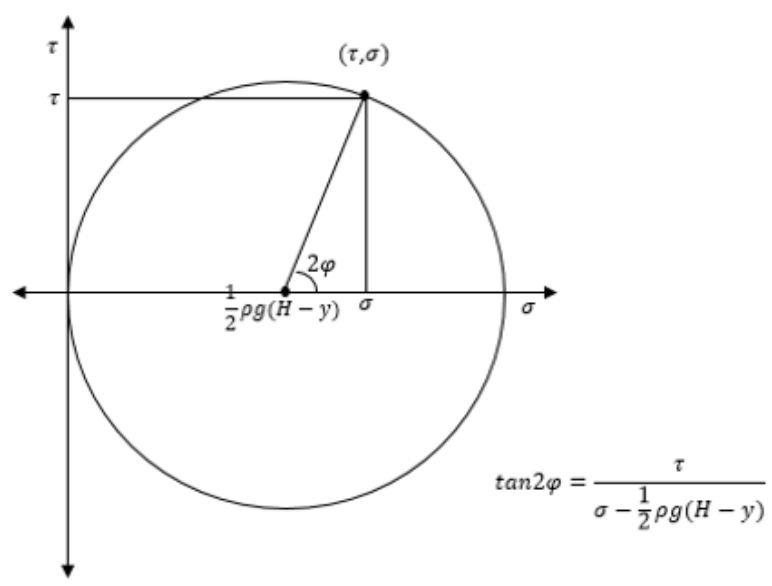

Figure 6. Mohr's Circle Equation 12

The fracture angle of this problem is,

$$
\tan \tan 2 \varphi=\frac{\tau}{\sigma-\frac{1}{2} \rho g(H-y)}
$$

Since of $\sigma_{\min }$ is in direction of $x$ axis, this will simplify the calculus because :

$$
\tan \tan \varphi=\frac{d y}{d x}
$$

Where $\frac{d y}{d x}$ is the slope direction of curving fracture line

If the value of $\mathrm{y}$ in equation $12 \mathrm{a}$ to $\mathrm{d}$ is different, then the magnitude of the Mohr circle will also be different (Figure 7).

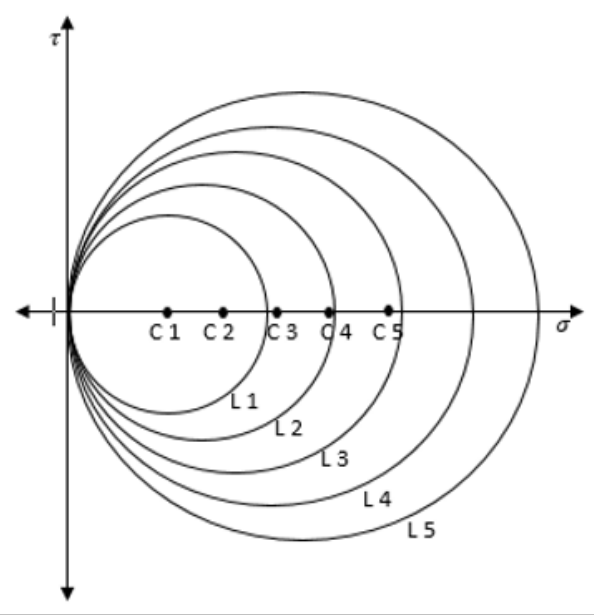

Figure 7. Mohr's Circle Equation 12 for Several $y$ Values

\subsection{Applying The Coulomb Fracture Criterion $\tau-\sigma$}

Before the model was considered, the analysis of the curving fracture (Figure 1 and 2) show that: When $\sigma$ is small, the position is near the surface, the fracture angle $\varphi$ is high, goes to $\frac{\pi}{2}$ and, when $\sigma$ is large, the position is around the bottom, the fracture angle $\varphi$ is low

By substituted the conditions above into the Mohr's equation (Equation 1), the Mohr's circle (Figure 4) became a half parabola curve $\tau-\sigma$ as it is shown in Equation 12.

$$
\tau=A \sigma^{\frac{1}{2}}
$$

The form of the above equation compatible with the Mohr Circle which contains $\sigma^{2}$ and $\tau^{2}$ as shown in Figure 7. Where the coefficient $\mathrm{A}$ can be interpreted as a friction coefficient in the terms of $\sigma^{\frac{1}{2}}$ or $A=\frac{d \tau}{d \sigma^{\frac{1}{2}}}$.

\subsection{Intercepting The Mohr Circle with The $\tau-\sigma$ Model}

Figure 9 is showing how the intersection between the $\tau-\sigma$ and the Mohr circle.

The $\tau-\sigma$ curve intersects at two points and can be seen in Figure 10 below:

1. The origin where it gives $2 \varphi^{*}=\pi \rightarrow \varphi^{*}=\frac{\pi}{2}$. This is the fracture along $\mathrm{y}$ or the vertical fracture plane that cause slide $\mathrm{dx}$ collapses

2.The point where gives $2 \varphi<\pi$ or $<\varphi \frac{\pi}{2}$. This is the fracture at the bottom which will continuously from curving fracture plane.

In this step the $\tau=A \sigma^{\frac{1}{2}}$ is used for calculation of producing $\varphi$ and then $\frac{d y}{d x}$ to get the curve equation. Substituting equation 


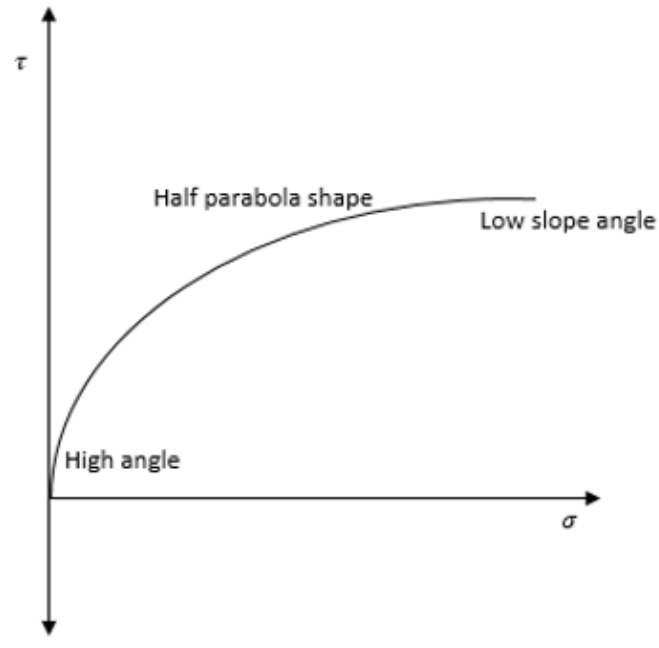

Figure 8. A Sketch of $\tau-\sigma$ Model Equation

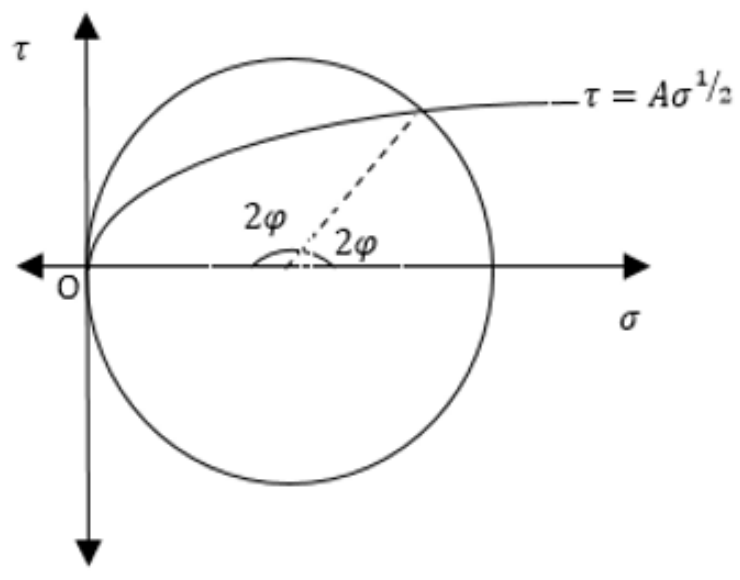

Figure 9. Intersection Between $\tau-\sigma$ Curve and Mohr Circle

13 into 12 gives value $\sigma$ in Equation 14 and $\tau$ in Equation 15:

$$
\begin{aligned}
& {\left[\sigma-\frac{1}{2} \rho g(H-y)\right]^{2}+A^{2} \sigma=\left[\frac{1}{2} \rho g(H-y)\right]^{2}} \\
& \sigma^{2}-\rho g(H-y) \sigma+A^{2} \sigma=0
\end{aligned}
$$

And since $\sigma$ is no always $(\sigma \neq 0)$, then:

$$
\sigma=\rho g(H-y)-A^{2}
$$

Then for $\tau$ :

$$
\tau=A \sigma^{\frac{1}{2}}=A\left[\rho g(H-y)-A^{2}\right]^{\frac{1}{2}}
$$

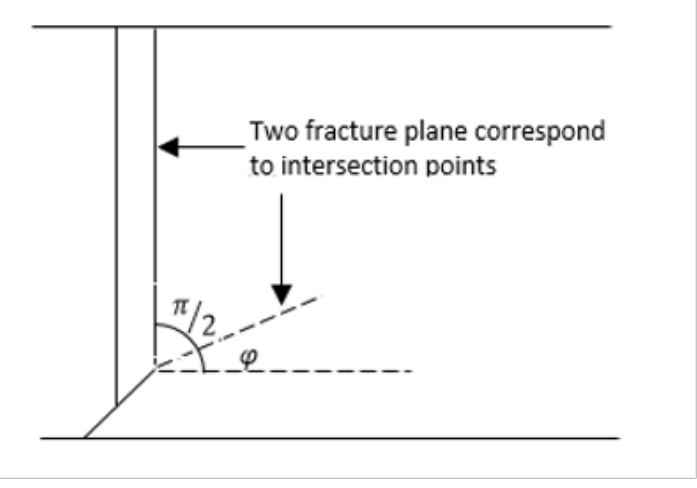

Figure 10. The Positions of Two Fracture Planes

From definition $\sigma$ and $\tau$ we can get the value of $\tan ^{2} \varphi$ in Equation 16 below:

$$
\begin{aligned}
& \tan 2 \varphi=\frac{\tau}{\sigma-\frac{1}{2} \rho g(H-y)}=\frac{A\left[\rho g(H-y)-A^{2}\right]^{\frac{1}{2}}}{\left[\frac{1}{2} \rho g(H-y)-A^{2}\right]} \\
& \tan 2 \varphi=\frac{A\left[\rho g(H-y)-A^{2}\right]^{\frac{1}{2}}}{\left[\rho g(H-y)-A^{2}-\frac{1}{2} \rho g(H-y)-A^{2}\right]}
\end{aligned}
$$

Trigonometry gives :

$$
\tan \tan 2 \varphi=\frac{2 \tan \varphi}{1-\tan ^{2} \varphi}
$$

Produces quadratic equation of :

$$
(\tan \tan 2 \varphi) \tan ^{2} \varphi+2 \tan \tan \varphi-\tan \tan 2 \varphi=0
$$

$$
\tan \tan \varphi_{12}=\frac{\sqrt[-2 \pm]{4+4 \tan ^{2} 2 \varphi}}{2 \tan \tan 2 \varphi}=\frac{\sqrt[-1 \pm]{1+\tan ^{2} 2 \varphi}}{\tan \tan 2 \varphi}
$$

If $\varphi$ goes to $\frac{\pi}{2}$ or $\mathrm{y}=\mathrm{H}$, and $\tau=\mathrm{A} \sigma^{\frac{1}{2}}=\mathrm{A}\left[\rho g(H-y)-A^{2}\right]^{2}$ has no physical meaning because if $\rho g(H-y)=0$ the terms under square roots are negative. The condition $\rho g(H-y)-$ $A^{2}=0$ gives restriction:

$$
\rho g(H-y)-A^{2} \geq 0, A^{2} \geq \rho h(H-y)
$$

Then, $y \leq H-\frac{A^{2}}{\rho g}$, the highest $\mathrm{y}$ that is following the curve. The calculation of $\varphi$ then becomes : 


$$
\begin{aligned}
\tan \tan \tan \varphi_{12} & =\left(-\frac{1}{\tan \tan 2 \varphi} \pm \sqrt{\frac{1}{\tan ^{2} 2 \varphi}}+1\right) \\
& =\frac{1}{\tan \tan 2 \varphi} \pm \sqrt{\frac{1+\tan ^{2} 2 \varphi}{\tan ^{2} 2 \varphi}} \\
& =-\frac{1}{\tan \tan 2 \varphi} \pm \sqrt{\frac{\sec ^{2} 2 \varphi}{\tan ^{2} 2 \varphi}} \\
& =\frac{1}{\tan \tan 2 \varphi} \pm \sqrt{\frac{1}{\cos ^{2} \varphi \frac{\sin ^{2} \varphi}{\cos ^{2} \varphi}}} \\
\tan \tan \varphi_{12} & =-\frac{1}{\tan \tan 2 \varphi} \pm \frac{1}{\sin \sin 2 \varphi} \\
\tan \tan \varphi_{1} & =-\frac{1}{\tan \tan 2 \varphi} \pm \frac{1}{\sin \sin 2 \varphi} \\
\tan \tan \varphi_{2} & =-\frac{1}{\tan \tan 2 \varphi}-\frac{1}{\sin \sin 2 \varphi}
\end{aligned}
$$

From the equation (4):

$$
\begin{aligned}
& \frac{1}{\tan \tan 2 \varphi}=\frac{\frac{1}{2} \rho g(H-y)-A^{2}}{A\left[\rho g(H-y)-A^{2}\right]^{\frac{1}{2}}} \\
& \sin \sin 2 \varphi=\frac{A\left[\rho g(H-y)-A^{2}\right]^{\frac{1}{2}}}{\sqrt{\left[A^{2}\left(\rho g(H-y)-A^{2}\right)\right]}+\left[\frac{1}{2} \rho g(H-y)-A^{2}\right]^{2}} \\
& \sin \sin 2 \varphi=\frac{\left[A^{2}\left(\rho g(H-y)-A^{4}\right)+\left[\frac{1}{2} \rho g(H-y)\right]^{2}-\rho g(H-y) A^{2}+A^{3}\right]^{\frac{1}{2}}}{\sin \sin 2 \varphi}=\frac{A\left[\rho g(H-y)-A^{2}\right]^{\frac{1}{2}}}{\frac{1}{2} \rho g(H-y)} \\
& \frac{1}{\sin \sin 2 \varphi}=\frac{\frac{1}{2} \rho g(H-y)}{A\left[\rho g(H-y)-A^{2}\right]^{\frac{1}{2}}}
\end{aligned}
$$$$
\tan \tan \varphi_{2}=-\frac{\left[\frac{1}{2} \rho g(H-y)-A^{2}\right]}{A\left[\rho g(H-y)-A^{2}\right]^{\frac{1}{2}}}+\frac{\frac{1}{2} \rho g(H-y)}{A\left[\rho g(H-y)-A^{2}\right]^{\frac{1}{2}}}
$$$$
=\frac{A}{\left[\rho g(H-y)-A^{2}\right]}>0,0<\varphi_{1}<\frac{n}{\pi} \text { applicable }
$$$$
\begin{aligned}
\tan \tan \varphi_{2} & =-\frac{\left[\frac{1}{2} \rho g(H-y)-A^{2}\right]}{A\left[\rho g(H-y)-A^{2}\right]^{\frac{1}{2}}}+\frac{\frac{1}{2} \rho g(H-y)}{A\left[\rho g(H-y)-A^{2}\right]^{\frac{1}{2}}} \\
& =\frac{-\left[\rho g(H-y)-A^{2}\right]}{A\left[\rho g(H-y)-A^{2}\right]^{\frac{1}{2}}}
\end{aligned}
$$

$$
=\frac{1}{A}\left[\rho g(H-y)-A^{2}\right]^{\frac{1}{2}}<0, \frac{\pi}{2}<\varphi, \pi \text { not applicable }
$$

2.6 Solution For $y(x)$ : The Curve of Sliding Plane for $\mathbf{0}<\varphi_{1}<\frac{\pi}{2}$ The solution $y(x)$ for curve sliding plane for $0<\varphi_{1}<\frac{\pi}{2}$ can be derived from equation 18 following mathematical procedure below:

$$
\begin{aligned}
\tan \tan \varphi_{1}=\frac{A}{\left[\rho g(H-y)-A^{2}\right]^{\frac{1}{2}}}=\frac{d y}{d x} \\
A d x=\left[\rho g(H-y)-A^{2}\right]^{\frac{1}{2} d y} \\
\text { let assume that } Z=\left[\rho g(H-y)-A^{2}\right] \\
d Z=-\rho g d y \\
A d x=-\frac{1}{\rho g} Z^{\frac{1}{2} d Z}
\end{aligned}
$$

$$
\begin{aligned}
&-A \rho g d x=Z^{\frac{1}{2} d Z} \\
&-A \rho g x+C_{1}=\frac{2}{3} Z^{\frac{1}{2}} \\
&-A \rho g x+C_{1}=\frac{2}{3}\left[\rho g(H-y)-A^{2}\right]^{\frac{3}{3}} \\
& x=0 \\
& y=0 \\
& C_{1}=\frac{2}{3}\left[\rho g(H-y)-A^{2}\right]^{\frac{3}{2}}, A^{2} \leq \sqrt{\rho g H} \\
&=-A \rho g x+\frac{2}{3}\left[\rho g(H-y)-A^{2}\right]^{\frac{3}{2}}-\frac{2}{3}\left[\rho g H-A^{2}\right]^{\frac{3}{2}} \\
& x=-\frac{2}{3 A \rho g}\left[\rho g(H-y)-A^{2}\right]^{\frac{3}{2}}+\frac{2}{3 A \rho g}\left[\rho g H-A^{2}\right]^{\frac{3}{2}}
\end{aligned}
$$

Using data $\left(X_{i}, Z_{i}\right)$ and chosen $H$ and $A$ with,

$$
y_{i} \leq H-\frac{A^{2}}{\rho g}, A^{2}<\sqrt{\rho g H}
$$

Using $\mathrm{y}$ as independent variable, $\mathrm{x}$ in equation 23 can be calculated and then the curve can be drawn (see illustrated in Figure 11)

Equation 10 can be used as theoretical reference for general curve fitting of the field data of $\left(x_{i}, y_{i}\right)$ by modifying into linear regression through the equation with variable:

$$
\begin{aligned}
W & =\alpha y+\beta \\
\frac{d x}{d y} & =\frac{1}{A}\left[\rho g(H-y)-A^{2}\right]^{\frac{1}{5}} \\
\left(\frac{d x}{d y}\right)^{2} & =-\frac{\rho g}{A^{2}} y+\frac{\rho g}{A^{2}} H-1
\end{aligned}
$$




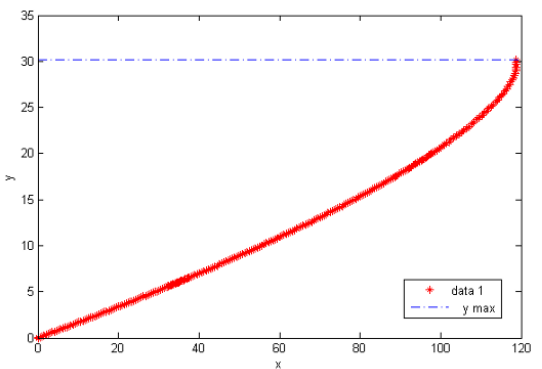

Figure 11. Relationship Between $y$ and $x$ in The 11 with Example $\mathrm{H}=30$ and $\mathrm{A}=250$

The linear plot is between $\left(\frac{d x}{d y}\right)^{2}$ vs $y$. Where $\alpha, \beta$ can be determining by this fitting as the slope and interception: From Equation 24 A can be estimated:

$$
\alpha=-\frac{\rho g}{A^{2}}, A=-\sqrt{\frac{\rho g}{\alpha}}
$$

From $\beta, H$ can be estimate:

$$
\beta=\frac{\rho g H}{A^{2}}-1, H=(\beta+1) \frac{A^{2}}{\rho g}
$$

Where $\mathrm{A}$ in equation 25 is the coefficient that appears in the near-collapse condition and $\mathrm{H}$ in equation 25 is height of the slope.

\subsection{Application of The Model In Evaluating Field Deformation After Sliding}

Since most place show curving sliding plane so that we should convince ourselves that the distribution of Coulomb failure at the curving plane should follow:

$$
\tau=A \sigma^{\frac{1}{2}}
$$
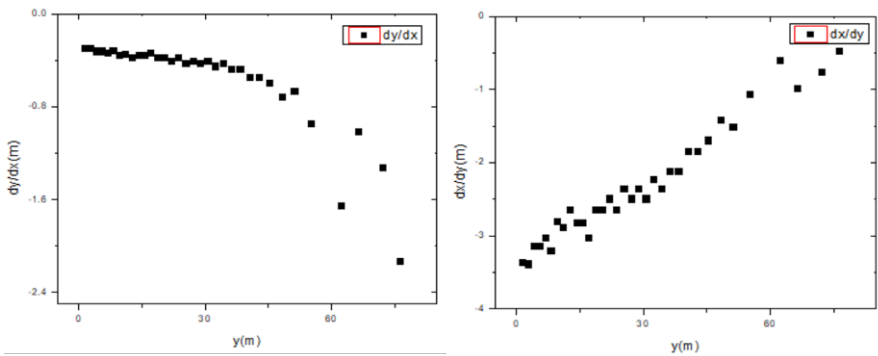

Figure 12. Example Data Preparation from $d y / d x$ Inverted Into $d x / d y$

In implementing the model, there a several stages as follows:

1. Determining position of vertical edge of the curving plane.

2. Make best estimate of density $\rho$ (almost liquefaction) from the material dropped left in the bottom plane. This can be also estimated from laboratory experiment.
3. Calculated $W=\left(\frac{d x}{d y}\right)^{2}$ from $\left(x_{1}, y_{i}\right)$ data and make sure that there is no $\frac{d y_{i}}{d x_{i}} \cong 0$ because will give very large value when it is inverted to be $\frac{d x}{d y}$ (see Figure 12).

4. Do linier regression fitting in $W$ versus $y$ data. The case below are the curve fitting applied in two landslide areas.

\section{DISCUSSION}

\subsection{Landslide Mechanism and Difficulty to Get Complete Data} Required by The Theory

Conclude from the above discussion that any curving sliding plane in believed coming from intersection between Mohr Circle of lithostatic maximum stress with $\tau-\sigma$ criterion of $\tau=A \sigma^{\frac{1}{2}}$. Is used intersecting $\tau-\sigma$ with Mohr Coulomb circles. Not like in the concept that Mohr circle is the envelope of triaxial measurement ( $\sigma$ is rising from a small value) (Heckston and Rutter, 2016; Liu, 2020). The collapse process with $\sigma_{\max }$ reaching $\rho g(H-y)$, it means Coulomb circle suddenly large and intersect $\tau-\sigma$, more over the $\tau-\sigma$ models $\tau=A \sigma^{\frac{1}{2}}$ from $(\tau=0, \sigma=0)$. Therefore this will give condition with two fracture angle $2 \varphi=\pi$ (intersection at origin) then produce $\varphi=\frac{\pi}{2}$ this the vertical angle of slicing rapture and $<\varphi<\frac{\pi}{2}$ that the angle cause curving sliding plane. Landslide occur in the heavy rainfall (Duc, 2013), no one has given a prediction before. The cliff would collapse and was sliding, so that no one witnessed the earth move. When realizing that happened so that it cannot get complete observed fact data.

\subsection{Meaning of Coefficient A Related to The Practical Knowledge of Sliding}

Coefficient $\mathrm{A}$ is the coefficient that appears in the near-collapse condition. A can be correlated to the loss of cohesion of the soil and water mixture on the cliff (Permanasari , 2017). Theoretically, the volume of mass mixture above the curved slip plane mixed with the volume of rainwater is what causes landslides. It should be noted that result $\mathrm{A}$ and the comparison between aggregate and water volume is one of the suggestions for early warning of collapse. In heavy rains that last for a long time, it is possible that the amount of water reaches a critical state which causes landslides.

\section{CONCLUSIONS}

Mathematical analysis using a special model $\tau-\sigma$ has been carried out to derive the collapse mechanism and derive an equation that proves the collapse is curved with the equation:

$$
x=\frac{2}{3 A \rho g}\left[\rho g(H-y)-A^{2}\right]^{\frac{3}{2}}+\frac{2}{3 A \rho g}\left[\rho g H-A^{2}\right]^{\frac{3}{2}}
$$

\section{ACKNOWLEDGEMENT}

Authors would like thank to earth and complex system laboratory ITB for facilities provided during writing this article.

\section{REFERENGES}

Alsubal, S., N. B. Sapari., I. S. Harahap, and M. A. M. Al-Bared (2019). A review on mechanism of rainwater in triggering landslide. In IOP Conference Series: Materials Science and Engineering, 513; 012009

Alvioli, M., F. Guzzetti, and M. Rossi (2014). Scaling properties of rainfall induced landslides predicted by a physically based model. Geomorphology, 213; 38-47 
Bin, Z, J., Xue, Y, X., Qi, H, S., tang, Y. J, and Zhun, B (2020). Mathematical statistical model of horizontal displacement and rainfall of step deformation landslide in three gorges reservoir area. Rock and Soil Mechanics, 41(1); 305-311

Dai, Z., Y. Huang., H. Cheng, and Q. Xu (2014). 3D numerical modeling using smoothed particle hydrodynamics of flow-like landslide propagation triggered by the 2008 Wenchuan earthquake. Engineering Geology, 180; 21-33

Das, B. M(2002). Principles of Geotechnical Engineering. Fifth edition, USA: Bill Stenquist

Das, B. M.(2016). Principles of Geotechnical Engineering, USA : Cemcage Learning

De-Blasio, F. V. (2011). Introduction to the physics of landslides: lecture notes on the dynamics of mass wasting. London: Springer Science \& Business Media

Duc, D. M. (2013). Rainfall-triggered large landslides on 15 December 2005 in Van Canh district, Binh Dinh province, Vietnam. Landslides, 10; 219-230

Fan, L., P. Lehmann., B. McArdell, and D. Or (2017). Linking rainfall-induced landslides with debris flows runout patterns towards catchment scale hazard assessment. Geomorphology, 280; $1-15$

Gariano, S. L. and F. Guzzetti (2016). Landslides in a changing climate. Earth-Science Reviews, 162; 227-252

Garry, B(2004). Understanding Soil Risk and Hazards, Nebraska: USDA

Greco, R., G. Giorgio., G. Capparelli, and P. Versace (2013). Early warning of rainfall-induced landslides based on empirical mobility function predictor. Engineering Geology, 153; 68-79

Heckson, A, and Rutter, E(2016). The Mohr-Coulomb criterion for intact rock strength and friction-a re-evaluation and consideration of failure under polyaxial stresses Solid Earth, 7; 493-508

Helwany, S(2007). Applied Soil Mechanics, USA: John Wiley

Labuz, J. P, and Zhang, A.(2012). Mohr Coulomb Failure Criterion, Rock Mech, 45:975-979

Lelovic, A, and Vasovic, D.(2020). Determination of Mohr-Coulomb Parameters for Modelling of Concrete, Crystals: 10, 808

Li, X., S. He., Y. Luo, and Y. Wu (2012). Simulation of the sliding process of Donghekou landslide triggered by the Wenchuan earthquake using a distinct element method. Environmental Earth Sciences, 65(4); 1049-1054

Liu, H (2020). Wellbore stability evaluation method based on the continuous tangent envelope of a Mohr circle. Science Progress,
301(1); 1-12

Murthy, V. N. S(2002). Geotechnical Engineering: Principles and Practices of Soil Mechanics and Foundation Engineering, Taylor and Francis Inc

Padmaja, S. B., G.V.N. Reddy, and Reddy, E. S (2021). Landslide stability analysis using mathematical approach. Material Today Proceedings, 1; 1-10

Permanasari, Vico, I, and Endi, E(2020). Determination of Slip Surface Using 2D Geoelectric Resistivity Method and Laboratory Analysis for Landslide Prone Area Pesawaran, Lampung Earth and Environmental Science, 537

Permanasari, Akabar, Handayani, Hendrajaya(2020). Determination of the type of soil using $2 \mathrm{~d}$ geoelectric method and laboratory analysis for landslide area Cililin west java Journal of Physics: Conference Series, 1127(1); 012041

Permanasari(2017). Mekanisme Runtuhan Tebing dengan Bidang Gelincir Melengkung Sebagai Awal Longsoran Lereng, Studi Kasus Daerah Cililin Kabupaten Bandung Barat. Dissertation: Unpublished

Saito, H., W. Murakami., H. Daimaru, and T. Oguchi (2017). Effect of forest clear-cutting on landslide occurrences: Analysis of rainfall thresholds at Mt. Ichifusa, Japan. Geomorphology, 276; 1-7

Shanmugam, G. and Y. Wang (2015). The landslide problem. Journal of Palaeogeography, 4(2); 109-166

Shi, C., Y. An., Q. Wu., Q. Liu, and Z. Cao (2016). Numerical simulation of landslide-generated waves using a soil-water coupling smoothed particle hydrodynamics model. Advances In Water Resources, 92; 130-141

Thomson, E, and Huppert, H(2007). Granular column collapses: further experimental results Journal Fluida, 575; 117-186

Wesley, L. D (2013). Fundamentals of Soil Mechanics for Sedimentary and Residual Soils, Jhon Wiley Press: Canada

Wu, L., Y. Zhou., P. Sun., J. Shi., G. Liu, and L. Bai (2017). Laboratory characterization of rainfall-induced loess slope failure. Catena, $150 ; 1-8$

Wu, L. Z., A. P. S. Selvadurai., L. M. Zhang., R. Q. Huang, and J. Huang (2016). Poro-mechanical coupling influences on potential for rainfall-induced shallow landslides in unsaturated soils. Advances In Water Resources, 98; 114-121

Zhang, L., X. Zhao., X. Yan, and X. Yang (2016). A semi-analytical method of stress-strain analysis of buried steel pipelines under submarine landslides. Applied Ocean Research, 59; 38-52 